\title{
An Optimization-Based Classification Approach with the Non-additive Measure
}

\author{
Nian Yan ${ }^{1}$, Zhengxin Chen ${ }^{1}$, Rong Liu ${ }^{2}$, and Yong Shi ${ }^{1,2}$ \\ ${ }^{1}$ College of Information Science and Technology, \\ University of Nebraska at Omaha, NE 68182, USA \\ \{nyan, zchen, yshi\}@mail.unomaha.edu \\ ${ }^{2}$ Chinese Academy of Sciences Research Center on Fictitious Economy and Data Science, \\ Graduate University of Chinese Academy of Sciences, Beijing 100080, China \\ \{liu.rong@163.com, yshi@guas.ac.cn\}
}

\begin{abstract}
Optimization-based classification approaches have well been used for decision making problems, such as classification in data mining. It considers that the contributions from all the attributes for the classification model equals to the joint individual contribution from each attribute. However, the impact from the interactions among attributes is ignored because of linearly or equally aggregation of attributes. Thus, we introduce the generalized Choquet integral with respect to the non-additive measure as the attributes aggregation tool to the optimization-based approaches in classification problem. Also, the boundary for classification is optimized in our proposed model compared with previous optimization-based models. The experimental result of two real life data sets shows the significant improvement of using the non-additive measure in data mining.
\end{abstract}

Keywords: Data Mining, Classification, Non-additive Measure, Optimization.

\section{Introduction}

In data mining, the classification is the task that aims to construct a model that could most efficiently distinguish different groups in a dataset. The optimization-based classification approaches formalize the data separation problem as the mathematical programming problems. Since Fisher's linear classification model [1], the groups are described as $\mathbf{A X} \pm b$, where $\mathbf{A}, \mathbf{X}, b$ are representing parameters to be learned, observations, and the constant boundary respectively. There are numerous optimizationbased classification models, from the classical linear classification to the popular Support Vector Machine (SVM). The common feature of these methods is the use of optimization techniques. Such technique as linear programming (LP) has already been widely used in the early studies of classification problem, e.g. Freed and Glover [2],[3] introduced two classification approaches based on the idea of reducing the misclassification through minimizing the overlaps or maximizing the distance of two objectives in a linear system, i.e. maximizing the minimum distances (MMD) of data from the critical boundary and minimizing the sum of the distances (MSD) of the data 
from the critical boundary. The linear SVM formulizes the bounding planes with soft margins to separate the data [4],[5].

An important indicator for the quality of the classifier is classification accuracy, as most existing researches on optimization-based classification approaches concern. Yet there are a number of other important issues for classification, such as speed, robustness, scalability, and interpretability [6]. Since interpretability refers to the level of understanding and insight that is provided by the classifier or predictor, it is related to the important aspect of handling the data. Unfortunately, the classic linear optimization-based classification approaches are with weak interpretability because it only considers the contributions from the attributes towards the classification equal to the sum of contributions from each individual attribute. Even with non-linear approach, the attributes are equally treated in the modeling stage. When interactions exist among attributes, the classification model does not correctly interpret the nature of the data. Thus, in this paper, we introduce an optimization-based classification approach with respect to the non-additive measure which is able to identify the interaction among the attributes and improve the classification performance in both accuracy and interpretability.

\section{Non-additive Measures and Integrals}

The concept of non-additive measures (also referred as fuzzy measure theory) and the ways of aggregation, nonlinear integrals, were proposed in the 1970s and have been well developed [7],[8],[9]. Non-additive measures have been successfully used as a data aggregation tool for many applications such as information fusion, multiple regressions and classifications [9],[10],[11],[12].

\subsection{Definitions}

Let finite set $X=\left\{x_{1}, \ldots, x_{n}\right\}$ denote the attributes in a multidimensional data set. The non-additive measures are defined as following [8],[13]:

Definition 1. A non-additive measure $\mu$ defined on $X$ is a set function $\mu$ : $\mathscr{P}(X) \rightarrow[0, \infty)$ satisfying

(1) $\mu(\phi)=0$

(2) $\mu(E) \leq \mu(F)$ if $E \subseteq F$

The property (2) is called monotonicity.

$\mathscr{P}(X)$ denotes the power set of $X$, which means the set of all subsets of $X, \mu_{i}$ denote the values of set function $\mu$ and $i=1, \ldots, 2^{n}-1$.

Definition 2. A signed non-additive measure $\mu$ defined on $X$ is a set function $\mu$ : $\mathscr{P}(X) \rightarrow(-\infty, \infty)$ satisfying (1) in definition 1 .

\subsection{Nonlinear Integrals}

Nonlinear integrals are regarded as the methods of aggregating $\mu_{i}$ in the set function $\mu$. The studies of non-additive measures and the corresponding nonlinear integrals could be 
found in the literatures [7],[8],[9],[13] from additive Lebesgue-like integral to the classic nonlinear integrals, i.e. Sugeno integral and Choquet integral. The Lebesgue-like integral is exactly the weighted sum of all the attributes and is widely used in forms of the linear models. However, considering the nonlinear relationships particularly the interactions among attributes, Sugeno integral and Choquet integral are the necessary data aggregation tools to be applied. Choquet integral is more appropriate to be chosen for data mining applications because it provides very important information in the interaction among attributes in the database compared with Sugeno integral [9]. Thus, in this paper, we choose the Choquet integral as the representation of the non-additive measure. Now let the values of $f,\left\{f\left(x_{1}\right), f\left(x_{2}\right), \ldots, f\left(x_{n}\right)\right\}$, denote the values of each attribute in the data set; let $\mu$ be a non-additive measure. The general definition of Choquet integral, with function $f: X \rightarrow(-\infty,+\infty)$, based on signed non-additive measure $\mu$ is defined by the formula (1)

$$
\text { (c) } \int f d u=\int_{-\infty}^{0}\left[\mu\left(F_{\alpha}\right)-\mu(X)\right] d \alpha+\int_{0}^{+\infty} \mu\left(F_{\alpha}\right) d \alpha
$$

Where $F_{\alpha}=\{x \mid f(x) \geq \alpha\}$ for $\alpha \in[0, \infty)$ and is called $\alpha$-cut set of $f$, for $\alpha \in[0, \infty), n$ is the number of attributes in the database.

When $\mu$ is additive, it coincides with the Lebesgue-like integral. Therefore, the Lebesgue-like integral is a special case of Choquet integral. The linear relationship of the attributes is still able to be identified when Choquet integral is used in data modeling. The general algorithm used to calculate the Choquet integral is shown below.

\section{General algorithm for calculating Choquet integral}

Step 1: Let $f=\left\{f\left(x_{1}\right), f\left(x_{2}\right), \ldots, f\left(x_{n}\right)\right\}$ denote the weighted values of each attribute for one given record. Then, we rearrange those values into a non-decreasing order:

$$
f^{*}=\left\{f\left(x_{1}^{*}\right), f\left(x_{2}^{*}\right), \ldots, f\left(x_{n}^{*}\right)\right\},
$$

where $f\left(x_{1}^{*}\right) \leq f\left(x_{2}^{*}\right) \leq \ldots \leq f\left(x_{n}^{*}\right)$. The sequence of $\left(x_{1}^{*}, x_{2}^{*}, \ldots, x_{n}^{*}\right)$ is one of the possibilities from the permutation of $\left(x_{1}, x_{2}, \ldots, x_{n}\right)$.

Step 2: Create the variables of $\mu$, where $\mu=\left\{\mu_{1}, \mu_{2}, \ldots, \mu_{2^{n}}\right\}$, and $\mu_{1}=\mu(\phi)=0$. Each of them represents the interaction of several attributes, e.g. $\mu_{2}=\mu\left(\left\{x_{2}^{*}, x_{3}^{*}\right\}\right)$.

Step 3: The value of the Choquet integral is calculated by:

(c) $\int f d \mu=\sum_{i=1}^{n}\left[f\left(x_{i}^{*}\right)-f\left(x_{i-1}^{*}\right)\right] \times \mu\left(\left\{x_{i}^{*}, x_{i+1}^{*}, \ldots, x_{n}^{*}\right\}\right)$, where $f\left(x_{0}^{*}\right)=0$.

The above 3-step algorithm is easy to understand but hard to implement with computer program. In this paper, we apply and modify the method proposed in [11] to calculating the Choquet integral. The method is illustrated in formula (2) and (3):

$$
\text { (c) } \int f d \mu=\sum_{j=1}^{2^{n}-1} z_{j} \mu_{j}
$$




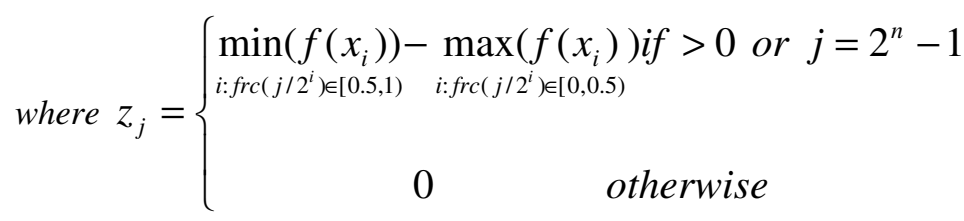

$\operatorname{frc}\left(j / 2^{i}\right)$ is the fractional part of $j / 2^{i}$ and the maximum operation on the empty set is zero. Also the criteria of judging $i$ equals to the following, if we transform $j$ into binary form $j_{n} j_{n-1} \ldots j_{1}$

$$
\begin{aligned}
& \left\{i \mid \operatorname{frc}\left(j / 2^{i}\right) \in[0.5,1)\right\}=\left\{i \mid j_{i}=1\right\} \text { and } \\
& \left\{i \mid \operatorname{frc}\left(j / 2^{i}\right) \in[0,0.5)\right\}=\left\{i \mid j_{i}=0\right\}
\end{aligned}
$$

Generally, the Choquet integral does not satisfy the additivity, which is defined as:

$(c) \int g d \mu+(c) \int f d \mu=(c) \int(g+f) d \mu, \mathrm{g}$ and $\mathrm{f}$ are functions defined as $f, g: X \rightarrow(-\infty,+\infty)$

From the above two versions of algorithms to the Choquet integral according to the general definition in formula (1), we observe that the pre-ordering of the values of attributes are required in both versions. However, the order of the attributes is sometimes only with only one ordering situation because different attributes may have different levels of scales. Thus, data normalization is needed to map the data into the same scale before the calculation of the Choquet integral. Two different types of data normalization are considered: the first is to perform the traditional data normalization process such as min-max normalization, z-score normalization and etc. [6]. In this way, each attribute is mapped into a certain range, e.g. $[0,1]$ for a typical min-max normalization. The real world situation is that you never know which data normalization is better for a given dataset unless to test all of them. The reason is that those different data normalization approaches treat every attributes equally. With this concern, the second type of data normalization is to set weights and bias on each attributes. In this way, we have to extend the definition of the Choquet integral, as follows:

$$
(c) \int(\mathbf{a}+\mathbf{b} f) d u
$$

where $\mathbf{a}=\left\{a_{1}, a_{2}, \ldots, a_{n}\right\}, \mathbf{b}=\left\{b_{1}, b_{2}, \ldots, b_{n}\right\}$ representing bias and weights to the attributes respectively.

However, with this scenario, $\mathbf{a}$ and $\mathbf{b}$ are not pre-determined and need to be learned during the training process of data mining. A typical searching algorithm such as genetic algorithm can be used to obtain those bias and weights according to the performance of the data mining task [11].

\section{Optimization-Based Classification Model with the Non-additive Measure}

In this section, we introduce the classical optimized-based classification models and extend the model with the signed non-additive measure. 


\subsection{Classical Optimization-Based Classification Models}

Freed and Glover [2],[3] introduced two classification approaches based on the idea of reducing the misclassification through minimizing the overlaps or maximizing the distance of two objectives in a linear system. The simple MSD classification model for two group classification is described as follows [2]:

Minimize $\sum_{i=1}^{m} \beta_{i}$

Subject to:

$$
\begin{aligned}
& y_{i}(A X-b) \leq \beta_{i} \\
& \beta_{i} \geq 0
\end{aligned}
$$

where $y_{i}=\{1,-1\}$ denotes the two different groups. The model is such a simple and efficient method that separates the data by searching a linear cut $A X$ and a suitable boundary $b$.

Before we introduce the use of signed non-additive measure in classification, the concept of separating data by hyperplanes is briefly described in section 3.2.

\subsection{Concept of Linearly Separable and SVM}

"Linearly separable" means that data is able to be perfectly separated by linear classification model. Data sets are sometimes linearly separable, most of the time not. The theorem of linearly separable is defined as: two groups of objects are linearly separable if and only if the optimal value of LP is zero. The proof of the theorem is from Bosch and Smith [14]. A linearly inseparable data set can not be perfectly separated by linear classification model due to the unreachable to the objective of LP.

The linear classification models contain such constrains as "AX" (or " $x^{T} w$ " in SVM). The efforts have been made to achieve the better separation in classification through constructing different objectives in optimization. For example, the very typical objective for SVM [4],[5] is

Minimize $\frac{1}{2}\|w\|^{2}+C \sum_{i}^{l} \xi_{i}$

Subject to:

$$
\begin{aligned}
& y_{i}(K(x, w)-b) \geq 1-\xi_{i} \\
& \xi_{i} \geq 0
\end{aligned}
$$

where $x_{i}$ denotes the parameters which need to be determined and are even possibly mapped into a even higher dimensional space by the kernel function $K\left(x_{i}, x_{j}\right)=\phi\left(x_{i}\right)^{T} \phi\left(x_{j}\right) . \phi: X \rightarrow H$ is a map from low dimension to high dimension, e.g. $\phi\left(x_{1}, x_{2}\right)=\left(x_{1}^{2}, \sqrt{2} x_{1} x_{2}, x_{2}^{2}\right)$ is a map from two dimensional to three dimensional space. $\mathrm{C}$ is a positive constant and is regarded as the penalty parameter. Given a linear kernel, the training procedure of SVM is to find linearly separating hyperplanes with 
the maximal margin in this higher dimensional space. The linear kernel function $K$, e.g. $K(x, w)=x^{T} w$, makes the SVM classification coincide with the linear classification models.

\subsection{Optimization-Based Non-additive Classification}

The optimization-based signed non-additive measure classification approach was developed by minimizing the SVM like objective and creating "Choquet hyperplanes" to separate the groups is as follows [15]:

$$
\text { Minimize } \frac{1}{2}\|\mu\|^{2}+C \sum_{i=1}^{m} \beta_{i}
$$

\section{Model 1}

Subject to:

$$
\begin{aligned}
& \left.y_{i}\left((c) \int f d \mu-b\right)\right) \leq \beta_{i} \\
& \beta_{i} \geq 0
\end{aligned}
$$

From programming perspective, the boundary value $b$ is hardly to be optimized because of the degeneracy issue of the programming. It is even much more difficult to solve with the above non-linear programming. The solution is to predetermine the value of $b$ or implement the learning scheme in the iterations, such as updating $b$ with the average of the lowest and largest predicted scores [10],[12]. The famous Platt's SMO algorithm [16] for SVM classifier utilized the similar idea by choosing the average of the lower and upper multipliers in the dual problem corresponding to the boundary parameter $b$ in the primal problem. Keerthi et al. [17] proposed the modified SMO with two boundary parameters to achieve a better and even faster solution.

The boundary $b$ in MSD becomes $b \pm 1$ in standard form of linear SVM, in which it is called the soft margin. The idea is to construct the separation belt in stead of a single cutting line. This variation of soft margin actually made the achievement that: the using of formation of $b \pm 1$ in optimization constrains coincidently solved the degeneracy issue in mathematical programming. The value of $b$ could be optimized by a simple linear programming technique. Thus we simplify the model in [12] into a linear programming solvable problem with optimized $b$ and non-additive measure with respect to generalized definition of the Choquet integral (formula 2, 3):

Minimize $C \sum_{i=1}^{m} \beta_{i}$

Subject to:

$$
\begin{aligned}
& \left.y_{i}\left((c) \int(\mathbf{a}+\mathbf{b} f) d \mu-b\right)\right) \leq 1+\beta_{i} \\
& \beta_{i} \geq 0
\end{aligned}
$$

In this model, the generalized "Choquet hyperplanes" separates the data with more flexibility and its geometric meaning could be found in [12]. We utilized the linear programming technique for solving the non-additive measure $\mu$. The parameters from $\mathbf{a}$ and $\mathbf{b}$ are optimized by genetic algorithm as we mentioned earlier. 


\section{Experimental Results}

We conduct the proposed approach (Model 2) on two datasets with comparisons to other popular approaches, i.e. Decision Tree (C4.5) and SVMs.

\subsection{US Credit Card Dataset}

The credit card dataset is obtained from a major US bank. The data set consists of 65 attributes and 5000 records. There are two groups: current customer (4185 records) and bankrupt customer ( 815 records). The task is to predict the risk of bankruptcy of customers. We regard the current customers as good customers and bankruptcy as bad. In order to reduce the curse of dimensionality, we use hierarchical Choquet integral [12],[18], that calculates the Choquet integral hierarchically, to Model 2 for decision makings on the new applicants. The way of hierarchical is determined by human experts. We use randomly sub-sampling 10 -fold cross-validation ( $90 \%$ for training and $10 \%$ for testing). The results are summarized in Table 1.

Table 1. Classification accuracy (\%) on US credit card dataset

\begin{tabular}{|c|c|c|c|}
\hline & $\begin{array}{l}\text { Sensitivity } \\
\text { (Good) }\end{array}$ & $\begin{array}{l}\text { Specificity } \\
\text { (Bad) }\end{array}$ & $\begin{array}{l}\text { Accuracy } \\
\text { (Overall) }\end{array}$ \\
\hline & \multicolumn{3}{|c|}{ Linear_SVM } \\
\hline Training & 69.8 & 87.2 & 78.5 \\
\hline Testing & 69.1 & 84.6 & 69.4 \\
\hline \multicolumn{4}{|c|}{ Polynomial_SVM } \\
\hline Training & 69.9 & 88.1 & 79.0 \\
\hline Testing & 68.7 & 85.5 & 69.1 \\
\hline \multicolumn{4}{|c|}{$R B F \_S V M$} \\
\hline Training & 71.5 & 88.4 & 80.0 \\
\hline Testing & 69.1 & 85.6 & 69.5 \\
\hline \multicolumn{4}{|c|}{ See5.0 (C4.5) } \\
\hline Training & 74.8 & 88.4 & 81.6 \\
\hline Testing & 69.5 & 81.8 & 69.8 \\
\hline \multicolumn{4}{|c|}{ Model 2} \\
\hline Training & 75.0 & 85.8 & 80.5 \\
\hline Testing & 70.9 & 81.6 & 71.1 \\
\hline
\end{tabular}

There is no such significant difference in terms of classification accuracy among those different approaches. Our approach performs best on testing dataset while See 5.0 performs best on training. One advantage of our approach is reliability which refers to as the classification model achieves similar accuracy on both training and testing dataset.

\subsection{UCI Liver Disorder Dataset}

The Liver Disorder dataset is obtained from UCI Machine Learning Repository (www.ics.uci.edu/ mlearn/MLRepository.html). This is a two-group classification problem and data consists of 6 attributes and 345 samples. We perform model 2 with 
Table 2. Classification accuracy (\%) on liver disorder dataset

\begin{tabular}{l|ll}
\hline \multicolumn{1}{c|}{ Classification Methods } & Training & Testing \\
\hline Linear_SVM & 63.9 & 55.6 \\
Polynomial_SVM & 65.1 & 60.0 \\
RBF_SVM & 100.0 & 75.4 \\
See5.0(C4.5) & 88.9 & 69.1 \\
Model 2 & 79.3 & 75.9 \\
\hline
\end{tabular}

a 10 -fold cross-validation (90\% for training and $10 \%$ for testing) on the dataset. The comparison results on average classification accuracy are summarized in Table 2.

We observe that the linear SVM performs worst that shows the linearly inseparable property of the dataset. The RBF SVM model classifies the dataset with $100 \%$ for training and $75.44 \%$ for testing. The proposed Model 2, which is based on the signed non-additive measure, performs $79.34 \%$ and $75.86 \%$ on training and testing respectively. This result is comparable to the RBF kernel SVM.

\section{Conclusions}

In this paper, we proposed a new optimization-based approach for classification when we believe the attributes have interactions. The new approach achieved higher accuracy of classification on two real life datasets compared with traditional approaches. We introduced the concept of non-additive measures as the data integration approach to optimization-based classification in data mining. The use of the Choquet integral with respect to the signed non-additive measure identified more hidden interactions among attributes and contributes to construct more reliable classification models. The learned non-additive measure shows some impact from the joint effect from several certain attributes towards classification and provides a potentially better interpretability of classification model. In the future, more experiments will be conducted for a better illustration both in classification performance and model representation by describing the relationship of learned $\mu$ and the interactions.

Acknowledgments. This research has been partially supported by a grant from National Natural Science Foundation of China (\#70621001, \#70531040, \#70501030, \#70472074), National Natural Science Foundation of Beijing \#9073020, 973 Project \#2004CB720103, National Technology Support Program \#2006BAF01A02, Ministry of Science and Technology, China, and BHP Billion Co., Australia.

\section{References}

1. Fisher, R.A.: The Use of Multiple Measurements in Taxonomic Problems. Annals of Eugenics 7, 179-188 (1936)

2. Freed, N., Glover, F.: Simple but powerful goal programming models for discriminant problems. European Journal of Operational Research 7, 44-60 (1981) 
3. Freed, N., Glover, F.: Evaluating alternative linear, programming models to solve the twogroup discriminant problem. Decision Science 17, 151-162 (1986)

4. Vapnik, V.: The Nature of Statistical Learning Theory. Springer, New York (1995)

5. Smola, A.J., Scholkopf, B.: A tutorial on support vector regression. Statistics and Computing 14, 199-222 (2004)

6. Han, J., Kamber, M.: Data Mining Concepts and Techniques, 2nd edn., pp. 286-289. Morgan Kaufmann Publishers, Inc., San Francisco (2002)

7. Choquet, G.: Theory of capacities. Annales de l'Institut Fourier 5, 131-295 (1954)

8. Wang, Z., Klir, G.J.: Fuzzy Measure Theory. Plenum, NewYork (1992)

9. Wang, Z., Leung, K.-S., Klir, G.J.: Applying fuzzy measures and nonlinear integrals in data mining. Fuzzy Sets and Systems 156(3), 371-380 (2005)

10. Xu, K., Wang, Z., Heng, P., Leung, K.: Classification by Nonlinear Integral Projections. IEEE Transactions on Fuzzy Systems 11(2), 187-201 (2003)

11. Wang, Z., Guo, H.: A New Genetic Algorithm for Nonlinear Multiregressions Based on Generalized Choquet Integrals. In: The Proc. of Fuzz/IEEE, pp. 819-821 (2003)

12. Yan, N., Wang, Z., Shi, Y., Chen, Z.: Nonlinear Classification by Linear Programming with Signed Fuzzy Measures. In: 2006 IEEE International Conference on Fuzzy Systems (July 2006)

13. Grabisch, M.: A new algorithm for identifying fuzzy measures and its application to pattern recognition. In: Proceedings of 1995 IEEE International Conference on Fuzzy Systems (March 1995)

14. Bosch, R.A., Smith, J.A.: Separating Hyperplanes and the Authorship of the Disputed Federalist Papers. American Mathematical Monthly 105(7), 601-608 (1998)

15. Yan, N., Wang, Z., Chen, Z.: Classification with Choquet Integral with Respect to Signed Non-Additive Measure. In: 2007 IEEE International Conference on Data Mining, Omaha, USA (October 2007)

16. Platt, J.C.: Fast Training of Support Vector Machines using Sequential Minimal Optimization, Microsoft Research (1998), http://research.microsoft.com/jplatt/ smo-book.pdf

17. Keerthi, S., Shevade, S., Bhattacharyya, C., Murthy, K.: Improvements to Platt's SMO algorithm for SVM classifier design. Tech Report, Dept. of CSA, Banglore, India (1999)

18. Murofushi, T., Sugeno, M., Fujimoto, K.: Separated hierarchical decomposition of the Choquet integral. International Journal of Uncertainty, Fuzziness and Knowledge-Based Systems 5(5) (1997) 